\title{
ATENCIÓN EDUCATIVA INCLUSIVA EN EL AULA. ESTUDIO DE CASO CON UN ALUMNO CON TRASTORNO GRAVE DE CONDUCTA
}

\section{Inclusive educational attention in the classroom. Case study with a student with challenging behaviour}

\author{
Albert Centellas Comellas \\ Universitat Autònoma de Barcelona. Facultat Ciènces de l'Educació. Departament de Peda- \\ gogía Aplicada. Plaça del Coneixement, edifici G6. 08193 Bellaterra. Cerdanyola del Vallés. \\ Barcelona \\ albertcentellas@gmail.com
}

Pedro JuRAdo DE LOS SANTOS

Universitat Autònoma de Barcelona. Facultat Ciènces de l'Educació. Departament de Pedagogía Aplicada

Recepción: 23 de noviembre de 2017

Aceptación definitiva: 16 de febrero de 2018

Resumen: Describimos el análisis del proceso de inclusión de un alumno con trastorno grave de conducta en un aula de educación primaria, desde la actuación docente y la dinámica que en el contexto ocurre, que se centra en las prácticas docentes con relación al proceso de aprendizaje, así como las barreras que afectan al proceso de aprendizaje. Se plantea el estudio de caso como el procedimiento óptimo para la consecución de los objetivos. La aplicación de la entrevista a los docentes, la utilización de datos eminentemente cualitativos y la correspondiente plasmación de resultados permite observar/ concluir que: las prácticas docentes se caracterizan por una actuación docente individualizada, carente de coordinación con otros agentes, orientada a la enseñanza; disfuncional con relación a los objetivos de aprendizaje; las actitudes hacia el trastorno grave de conducta se manifiestan mediante la justificación de falta de recursos para su atención, 
como un mecanismo retórico sobre la propia actuación; y el papel del contexto como consuetudinario en la satisfacción de necesidades educativas. Las barreras que afectan al aprendizaje se asocian al rol docente y al papel del contexto y organización educativa.

Palabras Clave: inclusión; barreras de aprendizaje; ética consecuencialista; coordinación docente: competencia docente.

AвsтRACT: We describe the analysis of the process of a student inclusion with challenging behaviour in a primary school classroom, from teaching performance and the dynamics that occur in the context, which focuses on teaching practices in relation to the process of student learning with challenging behaviour, as well as the barriers that affect the learning process. We considered the case study as the optimal procedure for achieving the objectives. The application of the interview to the teachers, the use of eminently qualitative data and the corresponding recording of results allows observing/concluding that: teaching practices are characterized by an individualized teaching performance, lacking coordination with other agents, oriented to teaching; dysfunctional in relation to learning objectives; attitudes towards the challenging behaviour are manifested through the justification of lack of resources for their attention, as a rhetorical mechanism on their own performance; and the role of context as customary in satisfying educational needs. The barriers that affect learning are associated with the teaching role and the role of the context and educational organization.

KEY WORDs: inclusion; learning barriers; consequentialist ethics; teaching coordination; teaching competence.

\section{Introducción}

$\mathrm{E}$ STE TRABAJO TIENE EL OBJETIVO DE ANALIZAR LAS PRÁCTICAS DOCENTES CON relación al proceso de aprendizaje del alumno con trastorno grave de conducta (TGC), así como las barreras que afectan al mismo y cómo intervienen los procesos organizativos del centro en su inclusión y su adaptación. Se plantea la conceptualización de TGC y su atención en los centros educativos de primaria, así como la percepción por parte del docente en relación a las prácticas que nos permiten determinar su concepción ética sobre su atención.

El manual DSM-V (APA, 2014) sitúa el trastorno de conducta (TC) dentro del grupo de trastornos destructivos del control de los impulsos y de la conducta; caracterizado en mostrar un patrón repetitivo y persistente de comportamiento en el que no se respetan los derechos básicos de otros, las normas y reglas sociales propias de la edad, lo que implica que estos cuadros conductuales requerirán de alguna manera atención multiprofesional.

El dictamen matiza que el alumno presenta desorganización conductual, tensión interna, impulsividad marcada, dificultades de aceptación de rutinas, límites y normas, baja tolerancia a la frustración con respuestas agresivas. Con lo que coincide en tres o más criterios del manual DSM-V (APA, 2014): 1- A menudo acosa, amenaza o intimida a otros; 2- A menudo inicia peleas; 11- A menudo miente para obtener 
objetos o favores, o para evitar obligaciones. Específicamente nos centramos en un TC infantil por el hecho de que el individuo muestra estos síntomas antes de cumplir los 10 años. Con una gravedad actual calificativa de Grave ya que dichos problemas conductuales provocan un daño considerable a los demás.

Asimismo, nos fijamos en la inclusión y su relevancia en los centros (Booth y Ainscow, 2011); en el TGC y su evolución y la gestión emocional de estos alumnos; en la competencia docente y su desarrollo (Perrenoud, 2012); en la ética docente y cómo esta se plasma en el aula a través de la actuación en la relación docente-discente (Howe y Miramontes, 2001), y en las barreras institucionales que afectan al alumno en su desarrollo a partir de tres aspectos: $10^{\circ}$ la cultura organizativa, $2 .^{\circ}$ la práctica docente y $3 .^{\circ}$ las características personales del alumno.

En este entramado, hay que tener presente cómo el docente gestiona el clima del aula teniendo presente la complejidad de la diversidad del aula, cómo organiza los recursos y de qué forma se percibe la coordinación con otros profesionales que intervienen en la atención a la diversidad.

\subsection{Objetivos}

La finalidad de nuestro estudio consiste, por una parte, en analizar las prácticas docentes que se realizan en el aula ordinaria y su adaptación al proceso de aprendizaje de un alumno con TGC. Específicamente, pretendemos analizar la competencia docente mediante las prácticas (estrategias didácticas utilizadas) que se adecuan al alumno con TGC e identificar los elementos sociales de aula que afectan al desarrollo del alumno. Por otra parte, valoraremos las barreras institucionales a la hora de atender al alumno con TGC; a nivel institucional analizaremos la presencia de principios inclusivos (presencia, participación y aprendizaje o logro) en la práctica docente de los agentes que intervienen con el alumno, así como la organización del centro con relación al proceso de enseñanza/aprendizaje, determinando la estructura relacional, coordinación docente y procesos de planificación.

\section{Método}

El trabajo se centra en un estudio de caso único (Stake, 2007), con el objeto de desarrollar una comprensión del fenómeno observado (Creswell, 2012), mediante observación naturalista, centrado en el sujeto y orientado al proceso en una realidad dinámica (Cook y Reichardt, 1995).

\subsection{Descripción del caso}

El caso que tratamos se refiere a un alumno, "J", de 11 años que cursa $5 .^{\circ}$ curso de Educación Primaria en un aula de 15 alumnos (60\% niñas y 40\% niños), dentro de un centro educativo de dos líneas con 254 alumnos, el 38\% de los cuales son de origen 
extranjero, y 42 maestros, en un contexto rural del interior de Cataluña, el núcleo poblacional es de unos 3500 habitantes aproximadamente. Actualmente presenta, según los informes psicopedagógicos, necesidades especiales derivadas de un trastorno emocional y de conducta.

En el historial de "J", destaca la atención por el servicio de psiquiatría del centro de salud mental infantil y juvenil (CSMIJ) a la edad de 6 años, en la que se inicia tratamiento farmacológico, por alteración de comportamiento relacionada con inestabilidad emocional en el aula y de relación con los compañeros; condicionándole llevar a cabo razonamientos (deductivos e inductivos); y mostrando conductas regresivas, heteroagresividad y dificultades adaptativas. Actualmente, "J" tiene un dictamen con un diagnóstico de TGC, con una disminución otorgada del 41\%.

El dictamen plantea los soportes que se plantean a nivel de centro durante el curso para atender sus necesidades mediante el Plan Individualizado, caracterizado por:

- Atención y seguimiento tutorial individualizado por parte del tutor.

- Soporte individual del maestro de Educación Especial a nivel emocional y personal.

- Atención en pequeño grupo en las áreas instrumentales.

- Soporte dentro del aula ordinaria para ayudarlo en la organización y en la actividad.

En el análisis de las diferentes dimensiones relacionadas, "J" muestra lo siguiente:

a) En la dimensión social, falta de habilidades en las estrategias sociales y de relación con los compañeros de aula y un estado de ánimo disarmónico. Estos elementos le producen malestar y dificultades en la adaptación a situaciones nuevas, manifestando actuaciones poco reflexionadas.

b) En la dimensión académica, se le diseña cada año el Plan Individualizado (PI) donde se priorizan la adecuación y adaptaciones metodológicas necesarias en las diferentes áreas del currículum. Recibe una atención individualizada en pequeño grupo fuera del aula y también un soporte interno de una hora en el aula por parte del maestro de educación especial (MEE), así como cinco horas en el aula con el auxiliar de educación especial (AEE). Partiendo de los informes y actas de evaluación, "J” presenta:

- Dificultades en la expresión de emociones y sentimientos a nivel oral y escrito.

- Dificultades en la adquisición de hábitos académicos en el aula y en el hogar.

- Dificultades en la adaptación a distintas situaciones socioacadémicas (trabajo en grupo, trabajo cooperativo, trabajo entre iguales...).

- Bajo nivel de concentración y atención ante las tareas académicas.

c) En la dimensión institucional y organizativa, el centro se caracteriza por la aplicación de estrategias de grupos flexibles a partir de 1. ${ }^{\circ}$ de Educación Primaria, separando el grupo en niveles en las áreas de lengua catalana y matemáticas; situando separadamente el grupo de alumnos de educación especial que es al que "J" asiste.

d) En la dimensión familiar, puede determinarse la existencia de problemas de cariz económico y situación de vulnerabilidad. El padre de "J" posee un diagnóstico de trastorno bipolar y regularmente está ingresado en un centro de salud mental. 


\section{ATENCIÓN EDUCATIVA INCLUSIVA EN EL AULA. ESTUDIO DE CASO CON UN ALUMNO CON TRASTORNO GRAVE DE CONDUCTA \\ ALBERT CENTELLAS COMELLAS Y PEDRO JURADO DE LOS SANTOS}

La madre, según los informes de los servicios sociales, tiene una actitud pasiva en la relación con los hijos. El hermano mayor de "J" va al instituto y la relación entre ellos se considera positiva.

\subsection{Instrumento}

Hemos considerado el acceso a la información a través de la entrevista, ya que permite acceder a aspectos más específicos de la reflexión de la persona, además permite ampliar las representaciones del docente (atribuciones, actitudes y valores) y la manera en que los propios alumnos entienden sus propias dificultades.

Las entrevistas se han diseñado atendiendo al estudio del caso y se han estructurado mediante 3 dimensiones: alumno, académica y actuación docente, de las cuales se derivan sus variables e indicadores correspondientes (ver Tabla 1).

\begin{tabular}{|c|c|c|c|c|c|c|c|c|}
\hline \multicolumn{9}{|c|}{$\begin{array}{l}\text { TABLA 1. Ejemplos de cuestiones realizadas en las entrevistas } \\
\qquad y \text { agentes de información }\end{array}$} \\
\hline & & & & \multicolumn{5}{|c|}{ Agentes* } \\
\hline Dimensión & Variable & Indicador & Ítem entrevista & $\mathrm{J}$ & $\mathrm{T}$ & $\mathrm{C}$ & M & A \\
\hline \multirow[t]{13}{*}{ Alumno } & \multirow[t]{5}{*}{ Autoconcepto } & \multirow{3}{*}{$\begin{array}{l}\text { 1. Percepción de las } \\
\text { propias dificultades }\end{array}$} & 1.1. ¿Cómo te describes a ti mismo? & $\mathrm{X}$ & & & & \\
\hline & & & 1.2. ¿En qué cosas no eres demasiado hábil? & $\mathrm{X}$ & & & & \\
\hline & & & $\begin{array}{l}\text { 1.3. ¿Cómo te sientes cuando las cosas no te } \\
\text { salen como te esperas? }\end{array}$ & $\mathrm{X}$ & & & & \\
\hline & & \multirow[t]{2}{*}{$\begin{array}{ll}\text { 2. } & \text { Percepción del } \\
& \text { futuro }\end{array}$} & $\begin{array}{l}\text { 2.2. ¿Hay alguna cosa que te preocupa en } \\
\text { relación al cambio al instituto? }\end{array}$ & $\mathrm{X}$ & & & & \\
\hline & & & 2.3. ¿Qué te gustaría ser de mayor? & $\mathrm{X}$ & & & & \\
\hline & \multirow[t]{7}{*}{$\begin{array}{l}\text { Adaptación } \\
\text { académica }\end{array}$} & \multirow{3}{*}{$\begin{array}{l}\text { 3. Percepción del } \\
\text { centro en relación } \\
\text { al desarrollo de su } \\
\text { rendimiento }\end{array}$} & $\begin{array}{l}\text { 3.1. ¿Estás contento con tus notas } \\
\text { académicas? }\end{array}$ & $\mathrm{X}$ & & & & \\
\hline & & & 3.2. ¿Crees que aprendes cosas en la escuela? & $\mathrm{X}$ & & & & \\
\hline & & & 3.3. ¿Te gusta ir a la escuela? & & & & & \\
\hline & & \multirow{2}{*}{$\begin{array}{l}\text { 4. Percepción del } \\
\text { soporte que recibe } \\
\text { dentro y fuera del } \\
\text { aula }\end{array}$} & $\begin{array}{l}\text { 4.1. ¿Te ayudan más dos maestros en el aula } \\
\text { que uno? }\end{array}$ & $\mathrm{X}$ & & & & \\
\hline & & & $\begin{array}{l}\text { 4.2. ¿Qué diferencias notas entre grupos } \\
\text { flexibles reducidos (catalán y matemáticas) y } \\
\text { dos maestros en el aula? }\end{array}$ & $\mathrm{X}$ & & & & \\
\hline & & \multirow{2}{*}{$\begin{array}{l}\text { 5. Valoración personal } \\
\text { de la relación } \\
\text { discente-docente }\end{array}$} & $\begin{array}{l}\text { 5.1. ¿Con algún maestro tienes una mejor } \\
\text { relación que con otros? }\end{array}$ & $\mathrm{X}$ & & & & \\
\hline & & & 5.2. ¿Cómo describes este maestro? & $\mathrm{X}$ & & & & \\
\hline & $\begin{array}{l}\text { Adaptación } \\
\text { social }\end{array}$ & $\begin{array}{l}\text { 6. Uso de estrategias } \\
\text { sociales }\end{array}$ & $\begin{array}{l}\text { 6.1. ¿Cómo valoras tu relación con los } \\
\text { compañeros de aula? }\end{array}$ & $\mathrm{X}$ & & & & \\
\hline
\end{tabular}

*J: Alumno; T: Tutor; C: Jefe de Estudios; M: Maestro Educación Especial; A: Auxiliar de Educación Especial. 
TABLA 1. Ejemplos de cuestiones realizadas en las entrevistas y agentes de información (cont.)

\begin{tabular}{|c|c|c|c|c|c|c|c|c|}
\hline & & & & \multicolumn{5}{|c|}{ Agentes* } \\
\hline Dimensión & Variable & Indicador & Ítem entrevista & $\mathrm{J}$ & $\mathrm{T}$ & $\mathrm{C}$ & M & $\mathrm{A}$ \\
\hline \multirow[t]{2}{*}{ Alumno } & & & $\begin{array}{l}\text { 6.2. ¿Te gustan más los trabajos solo o en } \\
\text { grupo? ¿Por qué? }\end{array}$ & $\mathrm{X}$ & & & & \\
\hline & & & $\begin{array}{l}\text { 6.3. ¿Consideras que tienes alguna dificultad } \\
\text { a la hora de hacer amigos? }\end{array}$ & $\mathrm{X}$ & & & & \\
\hline \multirow[t]{13}{*}{ Académica } & \multirow[t]{11}{*}{$\begin{array}{c}\text { Estrategias } \\
\text { metodológicas } \\
\text { inclusivas }\end{array}$} & \multirow[t]{2}{*}{$\begin{array}{l}\text { 7. Implica los alumnos } \\
\text { en su aprendizaje }\end{array}$} & $\begin{array}{l}\text { 7.1. ¿Cree que la organización de los } \\
\text { recursos del aula favorece un aprendizaje } \\
\text { autónomo? }\end{array}$ & & $\mathrm{X}$ & $\mathrm{X}$ & $\mathrm{X}$ & \\
\hline & & & $\begin{array}{l}\text { 7.2. ¿Se identifican los intereses de los } \\
\text { alumnos para construir el currículum? }\end{array}$ & & $\mathrm{X}$ & $\mathrm{X}$ & $\mathrm{X}$ & \\
\hline & & \multirow{3}{*}{$\begin{array}{l}\text { 8. La diferencia como } \\
\text { un recurso de } \\
\text { aprendizaje }\end{array}$} & $\begin{array}{l}\text { 8.1. ¿En qué criterios te basas a la hora de } \\
\text { diseñar grupos de trabajo? }\end{array}$ & & $\mathrm{X}$ & & & \\
\hline & & & $\begin{array}{l}\text { 8.2. ¿Los alumnos que tienen más } \\
\text { conocimientos o habilidades tutorizan a } \\
\text { alumnos con menos habilidad? }\end{array}$ & & $\mathrm{X}$ & & $\mathrm{X}$ & \\
\hline & & & $\begin{array}{l}\text { 8.3. ¿Se le proporcionan estrategias de control } \\
\text { emocional, habilidades sociales...? }\end{array}$ & & $\mathrm{X}$ & & $\mathrm{X}$ & \\
\hline & & \multirow[t]{3}{*}{$\begin{array}{l}\text { 9. La docencia } \\
\text { compartida }\end{array}$} & $\begin{array}{l}\text { 9.1. ¿El profesorado comparte esquemas de } \\
\text { programación? }\end{array}$ & & $\mathrm{X}$ & $\mathrm{X}$ & $\mathrm{X}$ & \\
\hline & & & $\begin{array}{l}\text { 9.2. ¿El profesorado se reúne para resolver } \\
\text { problemas conjuntamente cuando es motivo } \\
\text { de preocupación? }\end{array}$ & & $\mathrm{X}$ & $\mathrm{X}$ & $\mathrm{X}$ & $\mathrm{X}$ \\
\hline & & & $\begin{array}{l}\text { 9.3. ¿Crees que dos maestros en el aula } \\
\text { constituyen un modelo colaborador para el } \\
\text { alumnado? }\end{array}$ & & $\mathrm{X}$ & $\mathrm{X}$ & $\mathrm{X}$ & \\
\hline & & \multirow[t]{3}{*}{ 10. La evaluación } & $\begin{array}{l}\text { 10.1. ¿Se ha modificado algún criterio de } \\
\text { evaluación? }\end{array}$ & & $\mathrm{X}$ & $\mathrm{X}$ & $\mathrm{X}$ & \\
\hline & & & $\begin{array}{l}\text { 10.2. ¿Qué estrategias se utilizan para } \\
\text { evaluar las competencias de los alumnos? }\end{array}$ & & $\mathrm{X}$ & $\mathrm{X}$ & $\mathrm{X}$ & \\
\hline & & & $\begin{array}{l}\text { 10.3. ¿Los resultados son utilizados como } \\
\text { herramienta de ajuste a las necesidades del } \\
\text { alumno? }\end{array}$ & & $\mathrm{X}$ & & $\mathrm{X}$ & \\
\hline & \multirow[t]{2}{*}{$\begin{array}{l}\text { Recursos } \\
\text { materiales / } \\
\text { humanos }\end{array}$} & \multirow[t]{2}{*}{$\begin{array}{l}\text { 11. Distribución de los } \\
\text { recursos del centro }\end{array}$} & $\begin{array}{l}\text { 11.1. ¿Qué criterios se utilizan a la hora de } \\
\text { gestionar los recursos del centro en relación } \\
\text { al alumno con TGC? }\end{array}$ & & $\mathrm{X}$ & $\mathrm{X}$ & $\mathrm{X}$ & \\
\hline & & & $\begin{array}{l}\text { 11.2. ¿El equipo directivo facilita tiempo y } \\
\text { espacio para favorecer la coordinación entre } \\
\text { los profesionales? }\end{array}$ & & $\mathrm{X}$ & $\mathrm{X}$ & $\mathrm{X}$ & \\
\hline
\end{tabular}

*J: Alumno; T: Tutor; C: Jefe de Estudios; M: Maestro Educación Especial; A: Auxiliar de Educación Especial.

Ediciones Universidad de Salamanca / CC BY-NC-ND

Siglo Cero, vol. 49 (4), n. ${ }^{\circ}$ 268, 2018, octubre-diciembre, pp. 69-87 


\section{TABLA 1. Ejemplos de cuestiones realizadas en las entrevistas y agentes de información (cont.)}

\begin{tabular}{|c|c|c|c|c|c|c|c|c|}
\hline & & & & \multicolumn{5}{|c|}{ Agentes* } \\
\hline Dimensión & Variable & Indicador & Ítem entrevista & $\mathrm{J}$ & $\mathrm{T}$ & $\mathrm{C}$ & M & A \\
\hline \multirow[t]{14}{*}{$\begin{array}{l}\text { Ética } \\
\text { docente }\end{array}$} & \multirow[t]{7}{*}{$\begin{array}{l}\text { Presencia del } \\
\text { alumno }\end{array}$} & \multirow[t]{3}{*}{$\begin{array}{l}\text { 12. Dilemas éticos de la } \\
\text { profesión }\end{array}$} & $\begin{array}{l}\text { 12.1. ¿Cuál crees que es tu principal } \\
\text { finalidad en la escuela? }\end{array}$ & & $\mathrm{X}$ & $\mathrm{X}$ & $\mathrm{X}$ & $\mathrm{X}$ \\
\hline & & & $\begin{array}{l}\text { 12.2. ¿Qué entiendes por trastorno grave de } \\
\text { conducta? }\end{array}$ & & $\mathrm{X}$ & $\mathrm{X}$ & $\mathrm{X}$ & $\mathrm{X}$ \\
\hline & & & $\begin{array}{l}\text { 12.3. ¿Consideras que los alumnos con } \\
\text { problemas de conducta no deberían estar en } \\
\text { centros ordinarios? }\end{array}$ & & $\mathrm{X}$ & & $\mathrm{X}$ & \\
\hline & & \multirow{3}{*}{$\begin{array}{l}\text { 13. Relación pedagógica, } \\
\text { y comunicación en el } \\
\text { aula }\end{array}$} & 13.1. ¿Qué soluciones se han probado? & & $\mathrm{X}$ & $\mathrm{X}$ & $\mathrm{X}$ & $X$ \\
\hline & & & 13.2. ¿Cómo han funcionado? & & $\mathrm{X}$ & $\mathrm{X}$ & $\mathrm{X}$ & $X$ \\
\hline & & & $\begin{array}{l}13.3 \text { ¿El centro desarrolla algún programa de } \\
\text { modificación de conducta? }\end{array}$ & & $\mathrm{X}$ & $\mathrm{X}$ & $\mathrm{X}$ & \\
\hline & & $\begin{array}{l}\text { 14. Gestión de la } \\
\text { progresión de los } \\
\text { aprendizajes }\end{array}$ & $\begin{array}{l}\text { 14.1. ¿Qué tipo de coordinación hay entre } \\
\text { los profesionales y servicios que atienden al } \\
\text { alumno en relación a los aprendizajes? }\end{array}$ & & $\mathrm{X}$ & $\mathrm{X}$ & $\mathrm{X}$ & $\Lambda$ \\
\hline & \multirow[t]{2}{*}{$\begin{array}{l}\text { Participación } \\
\text { del alumno }\end{array}$} & \multirow[t]{2}{*}{$\begin{array}{l}\text { 15. Vínculos con teorías } \\
\text { inclusivas }\end{array}$} & $\begin{array}{l}\text { 15.1. ¿Parten de algún modelo inclusivo a la } \\
\text { hora de atender al alumno? }\end{array}$ & & $\mathrm{X}$ & $\mathrm{X}$ & $\mathrm{X}$ & $\mathrm{X}$ \\
\hline & & & $\begin{array}{l}\text { 15.2. ¿Cuáles son los criterios a la hora de } \\
\text { gestionar una conducta disruptiva en el aula } \\
\text { o centro? }\end{array}$ & & $\mathrm{X}$ & $\mathrm{X}$ & $\mathrm{X}$ & \\
\hline & \multirow[t]{5}{*}{$\begin{array}{l}\text { Colaboración } \\
\text { entre docentes }\end{array}$} & \multirow{5}{*}{$\begin{array}{l}\text { 16. Gestión y } \\
\text { orientación del } \\
\text { profesorado de } \\
\text { soporte }\end{array}$} & $\begin{array}{l}\text { 16.1. ¿Cómo se establece la coordinación } \\
\text { con el EAP? }\end{array}$ & & $\mathrm{X}$ & $\mathrm{X}$ & $\mathrm{X}$ & \\
\hline & & & $\begin{array}{l}\text { 16.2. ¿Qué orientaciones has recibido a la } \\
\text { hora de atender a un alumno con TGC? }\end{array}$ & & $\mathrm{X}$ & $\mathrm{X}$ & $\mathrm{X}$ & \\
\hline & & & $\begin{array}{l}\text { 16.3. ¿Has recibido alguna formación para } \\
\text { atender las conductas disruptivas del alumno? }\end{array}$ & & $\mathrm{X}$ & $\mathrm{X}$ & $\mathrm{X}$ & \\
\hline & & & $\begin{array}{l}\text { 16.4. ¿El maestro de soporte se ocupa } \\
\text { solamente de la participación del alumno } \\
\text { con TGC? }\end{array}$ & & $\mathrm{X}$ & & $\mathrm{X}$ & \\
\hline & & & $\begin{array}{l}\text { 16.5. ¿El centro tiene directrices claras de } \\
\text { cómo organizar el trabajo entre profesorado } \\
\text { de aula y soporte? }\end{array}$ & & $\mathrm{X}$ & $\mathrm{X}$ & $\mathrm{X}$ & \\
\hline
\end{tabular}

*J: Alumno; T: Tutor; C: Jefe de Estudios; M: Maestro Educación Especial; A: Auxiliar de Educación Especial.

\subsection{Procedimiento}

El proceso de investigación se inició con reuniones con la dirección del centro, el profesorado que atiende al alumno con TGC y las familias de la clase. Se acordó

Ediciones Universidad de Salamanca / CC BY-NC-ND

Siglo Cero, vol. 49 (4), n. ${ }^{\circ} 268,2018$, octubre-diciembre, pp. 69-87 
la temporización a la hora de realizar las entrevistas y se garantizó el anonimato del estudio. Se informó a las familias y docentes del proceso de investigación mediante una carta de presentación y una solicitud de aceptación del estudio.

A efectos del estudio nos hemos centrado en el alumno con TGC y en su contexto escolar. Los instrumentos se han aplicado teniendo presentes las normas éticas y deontológicas con los permisos, conocimiento y consentimiento de los implicados y de las familias en su caso. El registro de audio de las entrevistas ha sido transcrito para su posterior análisis.

La entrevista al alumno se realizó en un aula del propio colegio, de manera que se asegurara el contexto natural para conseguir más seguridad en el alumno. La entrevista fue individual para que el alumno pudiese expresarse libremente, evitando elementos que pudiesen influir en el sujeto. Se acordó que la entrevista se llevara a cabo sin que estuviese presente el tutor o el auxiliar.

Las entrevistas a los agentes que intervienen en el aula con el alumno se establecieron en un aula del interior del centro, de forma individual, con registro oral y realizando las anotaciones que se consideraban oportunas.

Las entrevistas planteadas permiten asegurar la información en busca de mayor grado de consistencia, cumpliendo el requisito de una triangulación por informantes (ver Tabla 2).

\begin{tabular}{|c|l|l|}
\hline \multicolumn{3}{|c|}{ TABLA 2. Instrumentos, objetivos e informantes en el proceso } \\
de recogida de datos
\end{tabular}

Todas las evidencias se organizaron mediante categorías y subcategorías (ver Tabla 3). En el proceso de recogida de datos se han obtenido un total de 302 unidades de registro o evidencias, representadas en frases o palabras de las entrevistas y/o del registro de aula; estas unidades de registro están explícitamente estructuradas en base a los agentes entrevistados.

Las relaciones establecidas entre las nueve categorías surgidas del proceso de categorización se muestran en la Figura 1, donde puede observarse la vinculación entre ellas y la consistencia para la interpretación de los resultados.

En este estudio, además de describir, comprender e interpretar los datos, no se pretende generar una teoría fundamentada con ellos, ya que no forma parte de nuestros objetivos, pero sí realizar conclusiones y discusión de estos. 


\begin{tabular}{|c|c|}
\hline Categoría & Subcategoría \\
\hline \multirow[t]{4}{*}{ 1. Conceptualización } & Factores individuales \\
\hline & Factores sociales \\
\hline & Factores familiares \\
\hline & Factores escolares \\
\hline \multirow[t]{3}{*}{ 2. Ética docente } & Ética docente \\
\hline & Perjuicios \\
\hline & Satisfacción en el centro \\
\hline \multirow[t]{3}{*}{ 3. Valores inclusivos } & Presencia \\
\hline & Participación \\
\hline & Aprendizaje \\
\hline \multirow[t]{7}{*}{ 4. Competencia docente } & La evaluación del discente \\
\hline & Plan Individualizado \\
\hline & Estrategias gestión emocional \\
\hline & Prácticas exitosas \\
\hline & Gestión clima de aula \\
\hline & Formación docente \\
\hline & Rol docente-discente \\
\hline \multirow[t]{3}{*}{ 5. Coordinación entre docentes } & Planificación docencia compartida \\
\hline & Actuación en el aula ordinaria \\
\hline & Planificación y seguimiento del discente \\
\hline \multirow[t]{4}{*}{ 6. Coordinación EAP } & Coordinación Tutor+EAP \\
\hline & Coordinación Tutor+MEE \\
\hline & Coordinación ED+Tutor+EAP+MEE \\
\hline & Coordinación AEE+EAP \\
\hline \multirow[t]{5}{*}{ 7. Organización del centro } & Grupos flexibles \\
\hline & Herramientas de evaluación de la institución \\
\hline & Gestión de recursos humanos \\
\hline & Gestión de recursos materiales \\
\hline & Gestión de las actitudes disruptivas \\
\hline \multirow[t]{3}{*}{ 8. Estrategias inclusivas } & Autoevaluación del discente \\
\hline & La diferencia como un recurso de E/A \\
\hline & Tutoría individualizada \\
\hline \multirow[t]{7}{*}{ 9. Alumno } & Estrategias sociales discente \\
\hline & Habilidades discentes \\
\hline & Autoimagen \\
\hline & Autoconcepto \\
\hline & Factores externalizantes \\
\hline & Factores neutrales \\
\hline & Factores internalizantes \\
\hline
\end{tabular}

Ediciones Universidad de Salamanca / CC BY-NC-ND

Siglo Cero, vol. 49 (4), n. ${ }^{\circ} 268,2018$, octubre-diciembre, pp. 69-87

$$
-77-
$$




\section{FIGURA 1. Vinculación de las categorías generales de la recogida de datos}

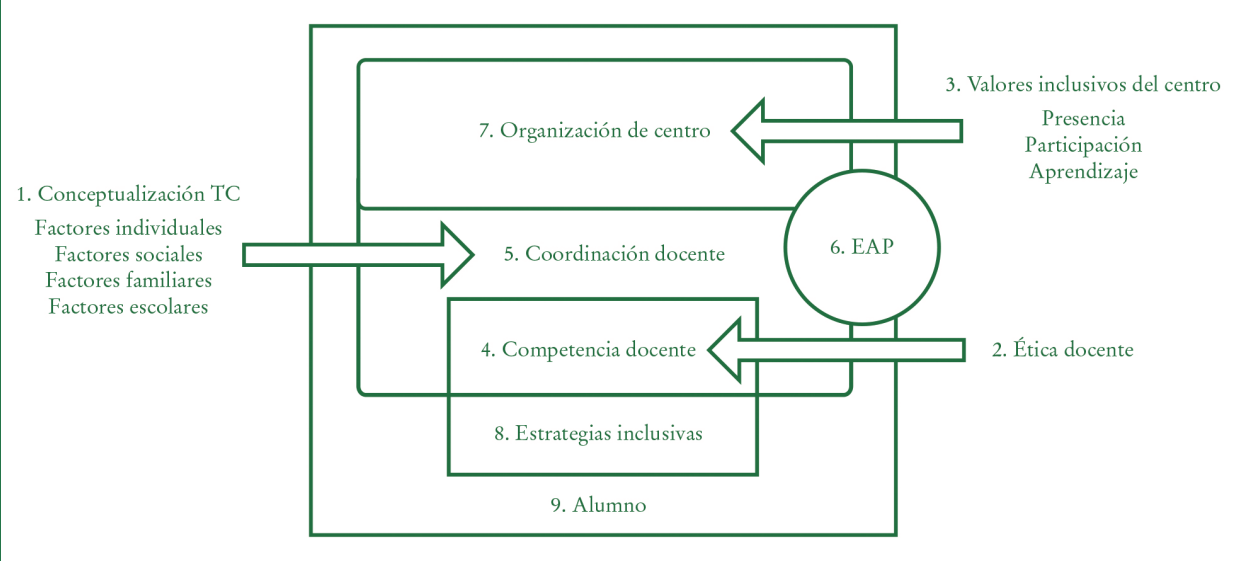

\section{Resultados}

Incidiremos en el análisis de la actuación docente, la planificación y la gestión, el proceso de inclusión y las barreras que influyen en el aprendizaje centrados en el caso objeto de análisis, a partir de las observaciones.

a) Planificación docente. La práctica docente con "J" no se plantea desde una planificación previa reflexionada de los objetivos y de las sesiones en el aula, lo que hace pensar en una estructura de docencia improvisada en algunas situaciones. La actuación docente en relación al alumno se realiza de manera individual y solitaria, sin coordinación con otros profesionales.

b) Ética docente. La actuación en el aula por parte de los profesionales se fundamenta en la propia experiencia para actuar y en el sentido común. La práctica, desde una consideración ética, no termina de favorecer el crecimiento personal de las personas tal y como la inclusión y la ética consecuencialista exigen. Se manifiesta un claro déficit de planificación, coordinación y reflexión docente de la propia práctica:

“Es un aspecto que tendría que hacer cada día y yo cada día no lo hago".

$$
\text { (E-Tutor) }
$$

"Las formas de atender a "J" en el centro son totalmente diferentes según el docente”.

$$
(\mathrm{E}-\mathrm{AEE})
$$

c) Seguimiento del proceso de aprendizaje: Plan Individualizado. El centro diseña el proceso de aprendizaje del alumno objeto de estudio mediante la herramienta 
del Plan Individualizado (PI), pero según los agentes entrevistados no se diseña a partir de proceso real evolutivo y académico del alumno.

“En la escuela se hace el PI con la directriz de elaborarlo dos cursos por debajo del curso actual del alumno, cuando el alumno posiblemente solo está un curso por debajo de algunas áreas, por ejemplo”.

$$
\text { (E-Tutor) }
$$

El PI es una herramienta en la que el tutor prácticamente no interviene y lo desarrolla el MEE, convirtiéndose, incluso, en una herramienta burocrática.

"El tutor prácticamente no interviene en el diseño del PI y el EAP únicamente dice si es necesario hacer PI o dictamen”.

d) Educación emocional. El centro evidencia una falta de plan de gestión de emociones de los alumnos, no existe ningún plan para evitar el malestar de los alumnos en relación a su proceso académico, responsabilizándose directamente de ello el tutor de aula.

"El centro se limita a transmitir contenidos para obtener buenos resultados en las pruebas internas y externas. Deja en segundo plano aspectos vitales como la educación emocional, el centro solo se basa en contenidos y resultados”.

$$
\text { (E-Tutor) }
$$

"Cuando tengo un conflicto no sé a quién explicarle, prefiero esperar al mediodía y explicarlo a mis padres, siempre me escuchan”.

e) Orientación tutor-discente. El proceso de orientación personal y social del alumno que se realiza en el centro es gestionado únicamente por el tutor.

“No puede ser que la culpa de todo lo que pasa en el aula sea de 'J', tenemos que cambiar la imagen que los otros perciben de ' $\mathrm{J}$ ' [...] es un grupo con unas relaciones intoxicadas y desde este aspecto he incidido mucho, mediante tutorías individualizadas mediante horas libres que tengo durante la semana”.

$$
\text { (E-Tutor) }
$$

f) Formación docente. Se manifiesta una clara falta de formación respecto el campo de estudio, el tutor se aferra únicamente a buscar herramientas de actuación según lo que se comenta en momentos informales con compañeros de la institución, y tanto MEE como AEE se aferran únicamente a la propia experiencia docente a la hora de actuar.

"Preparado no, a nivel de formación o a nivel de cursos no he realizado".

(E-Tutor)

"Al final te das cuenta que la propia experiencia es la que te va dando los recursos necesarios de cómo actuar y saber qué recursos utilizar y cuáles no”.

(E- MEE) 
"Sí, yo me siento preparado para alumnos con TC".

g) La evaluación del discente. Todos los agentes entrevistados consideran la evaluación desde una visión estrictamente curricular, no desde planteamientos competenciales. Se denota una falta de reflexión colectiva del proceso evaluativo del alumno.

"Se tiene que evaluar el día a día, con algún examen, pero no puede ser la nota final, tiene que ser la suma de todo”.

"El alumno te hará el examen, pero te lo hará peor de lo que podría. Primero porque lo aburre y segundo responde a voleo. [...] desconozco el proceso de evaluación del tutor en gran grupo y cómo estas se plantean a 'J'”.

\section{$(\mathrm{E}-\mathrm{MEE})$}

h) La diferencia como recurso de enseñanzalaprendizaje. En relación a las estrategias inclusivas, el tutor parte de la diferencia en el aula como un recurso positivo de enseñanza/aprendizaje y defiende la gestión de grupos "heterogéneos más que homogéneos" y que la diferencia hace el aprendizaje más global y compartido, pero se evidencia una falta de planificación para desarrollarlo mediante estrategias. Por ejemplo, la estrategia de la tutoría entre iguales no parte de ningún criterio a la hora de plantear las parejas.

"Se colocan más por afinidad que por niveles de aprendizaje".

$$
\text { (E-Tutor) }
$$

De esta forma la diferencia no se plantea como un recurso de mejora. El MEE plantea que el aprendizaje entre iguales es imposible con "J" y afirma "no haber visto la estrategia de la tutoría entre iguales en ningún momento y considera que 'J' no la aceptaría porque se sentiría inferior”.

Respecto el trabajo cooperativo, se manifiesta la importancia de la diversidad de roles en esta tipología de trabajo, pero de nuevo hay una falta de reflexión y planificación previa para dar respuesta a las situaciones que se dan durante la sesión en el aula. El tutor afirma:

"Creo que es una de las metodologías más interesantes a utilizar porque comparten un espacio en el que todos los alumnos aprenden entre ellos".

$$
\text { (E-Tutor) }
$$

i) Tutoría individualizada. La estrategia de la tutoría individualizada que se realiza docente tutor-discente una hora a la semana se ha diseñado y gestionado en todo momento por parte únicamente del tutor, sin la orientación de ningún otro agente del centro ni EAP. El tutor queriendo apostar por realizar un espacio semanal dedicado a la reflexión entre él y el alumno utiliza una de sus horas 
de trabajo personal para poder realizar y eso repercute en menos espacios de organización, planificación y diseños para su tarea diaria en el aula.

“Desde el primer momento consideré que el alumno necesitaba una orientación, pero no obtuve respuesta por parte del centro en relación al espacio y tiempo y decidí utilizar una hora de trabajo personal para realizarla”. En relación al diseño de esta afirma que "la planifico yo sin orientación por parte de EAP, Educación Especial, Equipo directivo...”.

(E-Tutor)

j) Factores externalizantes/internalizantes. Con relación a los elementos sociales del aula que influyen en el caso estudiado, el primer factor que condiciona las conductas de "J" en el aula es la propia competencia social y sus dificultades en la gestión emocional y de estrategias para interactuar entre iguales y con docentes. Los registros del aula evidencian una relación entre el grupo desigual y la relación de los compañeros con "J" es casi inexistente. Otro factor que interviene en el desarrollo social del alumno es que la orientación que está recibiendo en este ámbito de adquisición de estrategias sociales es insuficiente, ya que el propio alumno manifiesta dificultades para tener amigos.

"No sé hacer amigos y sin amigos no se pueden hacer los trabajos en grupo, por eso quiero estar solo".

Se evidencia una autoimagen negativa de él mismo en el aula y una clara necesidad de orientación de estrategias sociales entre iguales.

En relación a factores externalizantes que afecten a su conducta y la relación con los compañeros hay que atender a su falta de higiene y la poca puntualidad en la llegada al colegio.

k) Conceptualización/factores. Los agentes entrevistados, exceptuando el tutor, que señala la importancia de la escuela, atribuyen la aparición de conductas que se asocian al TGC únicamente a los factores individuales, sociales y familiares; obviando la importancia de los factores escolares en su crecimiento personal, social y académico.

Se evidencia un bajo rendimiento académico del alumno ocasionado por la desmotivación escolar debido a la no funcionalidad de los aprendizajes. Los datos afirman que el alumno adquiere un compromiso de aprendizaje cuando el objetivo y el diseño de la actividad son adaptados, estructurados y claros a lo largo de su desarrollo.

"Sí que me gusta aprender cosas nuevas, la informática me gusta mucho. En clase hago lo que me piden, lo hago y ya está. Pero me gustaría hacer más informática, se me da bien”.

1) Valores inclusivos: presencia, participación y aprendizaje. En relación a los principios inclusivos de presencia y participación, todos los agentes entrevistados en 
el estudio coinciden en poner en cuestión la escolaridad de cualquier alumno en un centro ordinario según los recursos de que se disponga.

"Él tiene que estar en una escuela ordinaria, a pesar de que no aprenda contenidos". (E-AEE)

"Cuando se llega a ciertos niveles de disfuncionalidad tanto a nivel de relación como cognitivo, pienso que lo ideal sería una jornada compartida en un centro de Educación Especial”.

(E-MEE)

En relación a la tipología de alumnos,

"Tienen un camino por recorrer, a lo largo de la escolaridad pueden cambiar todos, y bastante, pero dependerá de los recursos de que disponemos”.

(E-Tutor)

En ambos casos no hacen crítica de la propia gestión de los recursos y no ofrecen una visión clara de los principios inclusivos.

El alumno confirma en la entrevista que el sitio en el que se encuentra más cómodo es dentro del aula ordinaria y no cuando va en pequeño grupo a educación especial.

"Es cuando estoy en la clase con todos los alumnos que aprendo más y me siento mejor".

Para el MEE, que "J" vaya a un aula de educación especial fuera de su aula ordinaria es una práctica exitosa porque "así no molesta" y afirma que

"La posición del maestro de EE es privilegiada, yo tengo a 'J' en grupo reducido". El principio inclusivo de aprendizaje no es una prioridad para alumnos como "J". Por ejemplo, AEE culpa al alumno de la poca motivación,

"A él, el aprendizaje no le importa demasiado".

"Ya el año pasado estábamos observando que el aprendizaje no le motivaba, ni que le valores los resultados. Ahora lo que se hace es un trabajo conductual, reflexivo y que hable mucho, que exprese...”.

(E-MEE)

m) Planificación docencia compartida. En relación a la planificación de la docencia compartida y la coordinación, podemos destacar que todos los agentes implicados en el aula de "J" y entrevistados afirman que no han realizado ni un encuentro formal para planificar y reflexionar conjuntamente las sesiones y su rol en el aula con "J".

"Ni compartimos ni elaboramos ningún diseño de programación, ya sea en grupos flexibles, como en docencia compartida en el aula”.

(E-Tutor)

"No tenemos tiempo para coordinarnos, yo voy en el aula y hago lo que encuentro oportuno con 'J' y sus compañeros”.

(E-MEE)

Ediciones Universidad de Salamanca / CC BY-NC-ND

Siglo Cero, vol. 49 (4), n. ${ }^{\circ} 268,2018$, octubre-diciembre, pp. 69-87 
"No se ofrece ningún espacio en el horario para la coordinación entre MEE y Tutor porque no es factible en el horario”.

Hay discordancia entre el tutor y el MEE en relación a la docencia compartida. El tutor valida de forma positiva la estrategia de dos maestros en el aula, pero considera un problema que no haya coordinación en ningún momento con el MEE.

“Termino el curso sin haber conseguido una mejor gestión de la estrategia, ellos (MEE, AEE) continúan con un trato individualizado con 'J', no se plantean la estrategia hacia toda la diversidad".

$$
\text { (E-Tutor) }
$$

"La estrategia de la docencia compartida no funciona, porque cuando entro en el aula y el alumno detecta que solo estoy por él, éste no se deja ayudar”.

(E-E-MEE)

“En el centro no existe ninguna directriz de cómo debe ser la actuación en el aula de dos maestros, ni tampoco se ofrece ningún espacio para su coordinación, cosa que deberíamos mejorar”.

n) Planificación y seguimiento del discente. Queda de manifiesto, por otra parte, la necesidad de un replanteamiento organizativo para mejorar el asesoramiento al tutor y la coordinación entre profesionales, así como un sentido crítico respecto las agrupaciones flexibles.

"En ningún momento en todo el curso he tenido asesoramiento por parte del EAP, no ha habido ningún encuentro”.

$$
\text { (E-Tutor) }
$$

"Sí que se han reunido, pero no lo sé. En los casos más graves como es el suyo normalmente sí que hay encuentros”.

“EAP y Tutor sí se deben encontrar durante el curso ya que es necesario que el tutor reciba orientaciones al respecto”.

$(\mathrm{E}-\mathrm{MEE})$

“Te das cuenta de que para unos pocos les generas un estado emocional positivo y adecuado a ellos, pero para los alumnos de niveles más bajos es una metodología que no les permite crecer y les genera un estado emocional negativo afectando las relaciones entre ellos”.

$$
\text { (E-Tutor) }
$$


"No se tiene en cuenta una planificación de todos los docentes que actúan en grupos flexibles”.

$(\mathrm{E}-\mathrm{MEE})$

El centro no presenta ningún protocolo específico en relación a los alumnos con trastorno grave de conducta, y hacen uso de las NOFC (Normas de Organización y Funcionamiento de Centro) de forma común para todos los alumnos. Cada agente entrevistado establece una manera de actuar diferente en función de las actuaciones disruptivas de "J".

"Hay unas NOFC, pero con esa tipología de alumnado no funciona, el hecho de sancionar las conductas e imponer un castigo crispa mucho más su temperamento en el aula".

$$
\text { (E-Tutor) }
$$

"Que cuando hago tonterías me dejan sin patio, sin excursiones, salir fuera del aula. Cuando me castigan me siento mal, porque no estoy haciendo mi faena y estoy fuera, casi siempre estoy fuera...”.

n) Barreras institucionales. La situación descrita por el alumno nos da una evidencia clara de que la gestión de las conductas disruptivas se basa en la sanción y no en la reflexión, punición frente a prevención, a pesar de que el tutor manifiesta su compromiso para ofrecerle situaciones de intercambio.

Las barreras relacionadas con la cultura organizativa parten en primera instancia de la actitud reticente de los docentes ante la inclusión de estos alumnos, teniendo en cuenta los principios inclusivos analizados. Hay una tendencia a la desconfianza respecto de que todos los alumnos pueden aprender y cambiar. La coordinación y planificación docente también actúa de barrera, lo que lleva a que las soluciones se tomen de forma individualizada y sin implicar a todos los agentes educativos involucrados.

Hay una ausencia de responsabilidad compartida en el proceso educativo de "J", ya que los agentes entrevistados optan por responsabilizar al tutor, que no recibe orientación, ni seguimientos, ni plantean una tarea reflexionada conjunta de forma sistemática y sistémica.

"No hay reuniones con los maestros que atendemos a Miguel, solamente existen las sesiones de evaluación que hablamos de 38 alumnos en una hora y media, y momentos informales en el colegio".

$$
\text { (E-Tutor) }
$$

"No hay ningún protocolo en el centro de cómo actuar con esa tipología de alumnado, aplicamos un poco el sentido común de todos”. 


\section{Conclusiones y discusión}

Este trabajo contribuye a dar respuesta a una serie de interrogantes en torno a la figura del docente y su intervención en el aula en relación a un alumno con TGC, y cómo influye en ella la organización del centro, la coordinación docente y la actuación docente, desde una consideración ética, en relación al aprendizaje y la planificación para atender a la diversidad, específicamente a la inclusión de un alumno con TGC.

La figura docente y su práctica educativa con relación a “J” se caracteriza por un "bajo” nivel de realización de planificación de los objetivos de las sesiones y una gestión del aula disfuncional con relación a los objetivos desarrollados, lo que tiene consecuencias orientadas al desajuste en el aprendizaje de "J"; aspecto que desde una ética consecuencialista, fundamentada en la práctica educativa (Howe y Miramontes (2001), genera aprendizajes desadaptados en "J", considerándola, por tanto, como negativa. En general, esta práctica educativa implica una actuación individual y solitaria sin establecer coordinación, interdependencia con otros agentes educativos.

Un elemento que coincide con el estudio de Martín (2011), a nivel español, en educación primaria, es obviar el bienestar emocional del alumno en los centros, determinado por el desconocimiento por parte del docente en el uso de estrategias eficaces del manejo de la conducta; se observa ausencia de estrategias cooperativas, distribución del espacio inadecuado en el desarrollo de las actividades y falta de atención a los intereses y motivaciones del alumnado. Armas (2007) pone el énfasis en la relación entre alteraciones de conducta y su afectación de forma significativa con las relaciones de la persona en su medio social y en consecuencia de sus emociones, cosa que nos permite relacionar también en nuestro estudio la falta de estrategias de planificación, control y gestión emocional. No se diseñan actividades exitosas hacia el alumno, hay un factor de casualidad, y la evaluación se plantea desde una visión curricular explícitamente no competencial.

La relación docente-discente es disarmónica en el aula, pues cada profesional actúa de forma individual y en base a su criterio particular. Siguiendo las diez competencias de Perrenoud y Judit (2004), consideramos que el tutor debe plantearse una organización basada en estimular situaciones de aprendizaje y así implicar a los alumnos afectiva y efectivamente en las actividades educativas. La gestión de la progresión de los aprendizajes es una competencia clave para adquirir una visión longitudinal de los objetivos de enseñanza.

El centro no percibe la diferencia del alumnado como un reto y un potencial para la mejora de la calidad educativa; por ese motivo los separa por grupos homogéneos en algunas asignaturas. Hacer frente a la totalidad del grupo, practicando un soporte integral y desarrollando la cooperación entre alumnos a partir de una planificación y coordinación docente real, se establece como una necesidad para atender a todo el alumnado. En el contexto de centro, parece necesario, siguiendo a Perrenoud y Judit (2004), la participación docente en la gestión de la escuela, que se caracterice en organizar y hacer evolucionar dicha participación, así como coordinarla mediante un proyecto institucional colectivo y no únicamente del equipo directivo. 
Las conductas y elementos de aula que afectan positiva y negativamente en el desarrollo del alumno se basan en la visión del modelo del déficit, como podemos interpretar de los resultados, lo que conlleva repercusiones negativas en la autoimagen, autoconcepto y autoestima por parte del alumno.

Los factores internalizantes (Cooper y Upton, 1990) también van asociados a nuestro estudio, ya que "J" manifiesta una actitud agresiva con compañeros y profesorado fruto de las dificultades en las estrategias sociales, y asociada a pensamientos extraños y reiterativos que son difíciles de asimilar por los compañeros y le generan un rechazo de los iguales.

Teniendo presentes los principios inclusivos, siguiendo a Booth y Ainscow (2011), podemos afirmar que el centro educativo evidencia no cumplirlos, asumiendo desde la práctica argumentos segregadores. La planificación no se basa en la participación de los estudiantes en su aprendizaje y la comunidad no actúa de forma consensuada. Echeita (2006) ya describe la necesidad de promover experiencias de aprendizajes y socialización de todos los alumnos y entender la necesidad de crear espacios donde se respete y se viva la diversidad (Urbina, 2013). En relación al aprendizaje, el estudio destaca la falta de significatividad de los contenidos realizados en las sesiones, tal y como afirman Echeita y Cuevas (2011), no permite desarrollar la autonomía de aprendizaje en un futuro como herramienta competencial. Y, en relación a la participación del alumno, hay evidencias reiteradas de su desagrado cuando sale del aula, porque se siente apartado, lo que lleva a determinar que las decisiones no se fundamentan en las necesidades de "J", sino en las creencias del profesorado.

En relación a la organización de centro, no solo hemos de analizar las características de los alumnos como causas de sus dificultades (modelo del déficit), hace falta hacer un análisis de las barreras que existen en el contexto donde se encuentran estos alumnos y en donde interactúan (Echeita, 2006; Ainscow, Booth y Dyson, 2006; Booth y Ainscow, 2011; Martín, Sandoval y Simón, 2011) y estas pueden estar presentes en distintos niveles de la escuela (macro -escuela-, meso -ciclo superior-, micro -aula-), siendo necesario modificarlas. En general, coincidimos con Martín (2013) cuando manifiesta que una de las principales barreras en los centros educativos es la dificultad de coordinación entre docentes. Además, se le añade una ausencia de modelo de prevención de conflictos en el que se implique a toda la comunidad educativa y en donde adquiera protagonismo la educación emocional.

Desde el punto de vista de las limitaciones de este trabajo, debemos tener en cuenta que, aunque las entrevistas han ofrecido evidencias explícitas de la situación educativa del alumno con relación al docente y la institución escolar, se podría considerar aumentar las fuentes y técnicas de obtención de información con el objetivo de asegurar la consistencia de los resultados. Asimismo, la valoración de la competencia docente debería llevarnos a tener presentes otros elementos que afianzaran la información obtenida mediante la técnica de entrevista.

El estudio nos permite considerar nuevas líneas de trabajo basadas en diseñar un plan de soporte conductual positivo que, como señalan Canal y Martín (2002), plantea una evaluación funcional que vincula variables ambientales con las hipótesis relacionadas con la conducta problemática, en el caso de los TGC, orientados a diseñar 


\section{ATENCIÓN EDUCATIVA INCLUSIVA EN EL AULA. ESTUDIO DE CASO CON UN ALUMNO CON TRASTORNO GRAVE DE CONDUCTA \\ ALBERT CENTELLAS COMELLAS Y PEDRO JURADO DE LOS SANTOS}

protocolos de actuación que consideren la organización educativa y la intervención desde la inclusión.

La actuación docente frente un alumno con TGC no necesariamente se tiene que entender de forma equiparable a la que pueden ser actuaciones a otras necesidades. La práctica docente debe partir del alumno e ir incorporando evidencias de análisis y de contrastación para favorecer una atención basada en el alumno que promueva su aprendizaje y desarrollo.

\section{Referencias bibliográficas}

Ainscow, M., Воотн, T. y Dyson, A. (2006). Improving schools, developing inclusion. Londres: Routledge.

American Psychiatric Association (APA) (2014). Manual diagnóstico y estadístico de los trastornos mentales DSM-5. 5. ${ }^{a}$ ed. Madrid: Ed. Médica Panamericana.

Armas, M. (2007). Prevención e Intervención ante problemas de conducta. Estrategias para centros educativos y familias. Madrid: Wolters Kluwer.

Воотн, T. y Arnscow. M. (2011). Index for inclusion. Developing learning and participation in schools. Bristol: CESIE.

Canal, R. y Martín, M. V. (Coords.) (2002). Apoyo conductual positivo. Valladolid: Consejería de Sanidad y Bienestar Social. Recuperado de http://sid.usal.es/idocs/F8/FDO6644/ apoyo_conductual_positivo.pdf.

Cook, T. y ReIchardT, Ch. (1995). Métodos cualitativos y cuantitativos en investigación evaluativa. Madrid: Morata.

Cooper, P. y Upton, G. (1990). An ecosystemic approach to emotional and behavioural difficulties in schools. Educational Psychology, vol. 10, 4, 301-321.

Creswell, J. W. (2012). Educational Research. Planning, Conducting, and Evaluating Quantitative and Qualitative Research. Fourth Edition. Boston: Pearson Education, Inc.

EcheitA, G. (2006). Educación para la inclusión o educación sin exclusiones. Madrid: Narcea.

Echeita, G. y Cuevas, I. (2011). La educación inclusiva. En E. Martín y T. Mauri (Eds.), Orientación Educativa. Atención a la diversidad y educación inclusiva (pp. 11-27). Barcelona: Graó.

Howe, K. R. y Miramontes, O. B. (2001). La ética de la educación especial. Barcelona: Idea universitaria.

Martín Moreno, A. (2013). La situación del alumnado con problemas emocionales y de conducta en la comunidad de Madrid: Barreras y facilitadores para su inclusión educativa. Tesis doctoral. Madrid: Universidad Autónoma de Madrid.

Miles, M. B. y Huberman, A. M. (1994). Qualitative Data Analysis: an expanded sourcebook. Thousand Oaks, CA: SAGE Publication.

Perrenoud, P. (2012). Cuando la escuela pretende preparar para la vida. Barcelona: Graó.

Perrenoud, P. y Judit, A. (2004). Diez nuevas competencias para enseñar. Barcelona: Graó.

Simón, C., Sandoval, M. y Martín, A. (2011). Los problemas de comportamiento. En E. MarTín y T. MAuri (Coords.) Orientación educativa. Atención a la diversidad y educación inclusiva (pp. 147-167). Barcelona: Graó.

STAKe, R. E. (2007). Investigación con estudio de casos. Madrid: Morata.

Strauss, A., Corbin, J. y Zimmerman, E. (2002). Bases de la investigación cualitativa. Medellín, Colombia: Universidad de Antioquía.

URBINA, C. (2013). La compleja relación entre las concepciones sobre los procesos de inclusión y la práctica docente. Madrid: Universidad Autónoma de Madrid. 


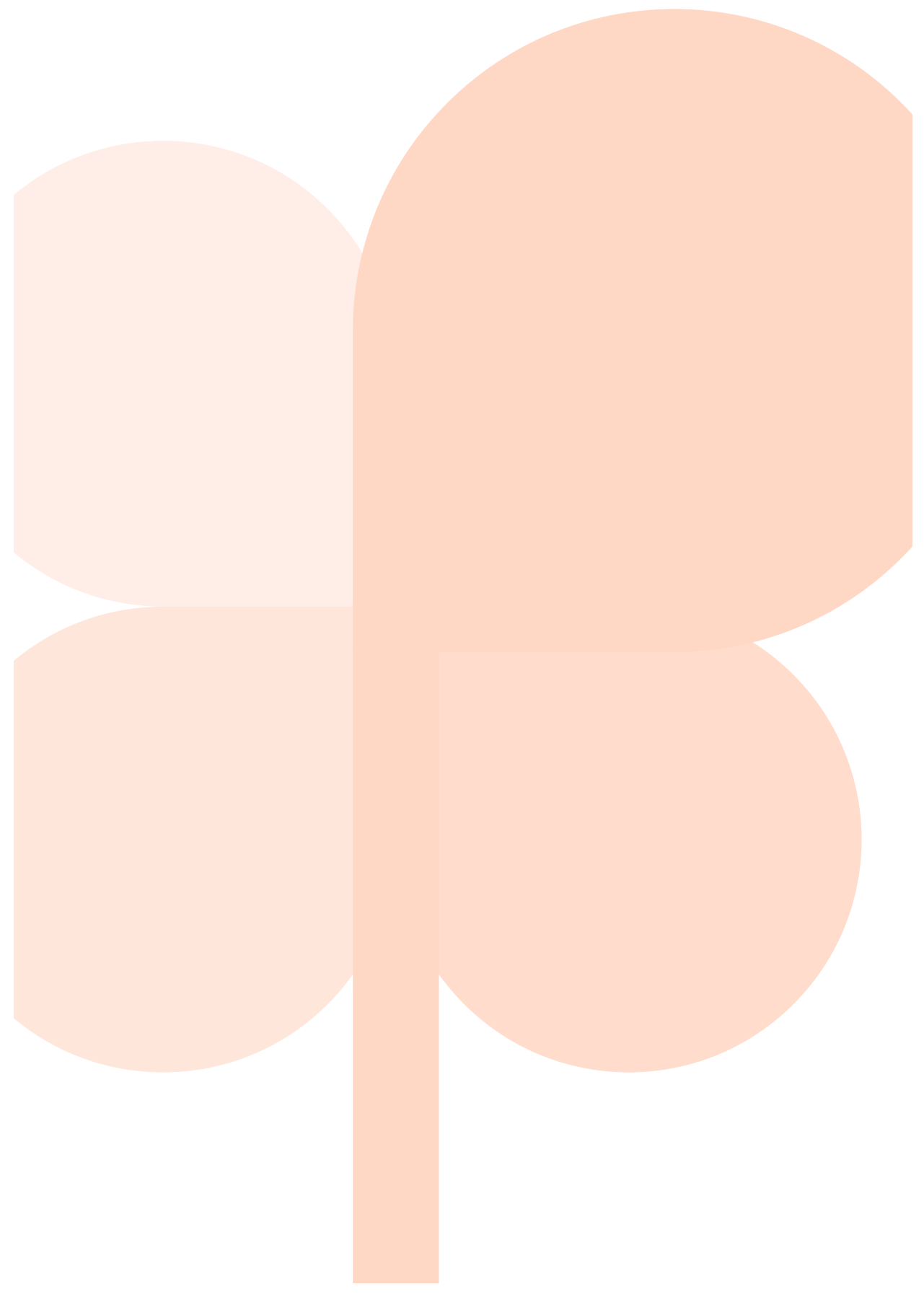

